\title{
Questes
}

\section{Compte rendu de la journée d'études « Le discours mythologique au Moyen Âge »}

\section{Sophie Albert}

\section{(2) OpenEdition}

\section{Journals}

\section{Édition électronique}

URL : http://journals.openedition.org/questes/1611

DOI : 10.4000/questes. 1611

ISSN : 2109-9472

Éditeur

Les Amis de Questes

\section{Édition imprimée}

Date de publication : 15 janvier 2006

Pagination : 83-92

ISSN : 2102-7188

\section{Référence électronique}

Sophie Albert, "Compte rendu de la journée d'études « Le discours mythologique au Moyen Âge » »,

Questes [En ligne], 8| 2006, mis en ligne le 01 janvier 2014, consulté le 23 septembre 2020. URL:

http://journals.openedition.org/questes/1611 ; DOI : https://doi.org/10.4000/questes.1611

Ce document a été généré automatiquement le 23 septembre 2020.

(c) Association des amis de «Questes » 


\title{
Compte rendu de la journée d'études « Le discours mythologique au Moyen Âge »
}

\author{
Sophie Albert
}

\section{RÉFÉRENCE}

« Le discours mythologique au Moyen Âge » organisée par le groupe de recherche

«Lectures médiévales » de l'équipe d'accueil «Littérature et herméneutique »

Toulouse, Université du Mirail, vendredi 13 janvier 2006

1 Florence Bouchet, après avoir souhaité la bienvenue aux assistants, commence par quelques mots de présentation. La journée d'étude reprend une tradition, un temps interrompue, d'organiser à l'Université du Mirail des rencontres de médiévistes. Le sujet choisi, «le discours mythologique », invite à repérer dans la littérature médiévale les permanences, les évolutions, les interprétations de la mythologie pré-chrétienne, dans une perspective résolument diachronique: les communications couvrent une large période allant $\mathrm{du} \mathrm{vI^{e }}$ au Xve siècle et inclut des textes latins et vernaculaires.

\section{Eléonore Andrieu, « L'art d'accommoder les mythes : présence et absence du discours mythologique dans I'histoire des rois francs ( $\mathrm{VI}^{\mathrm{e}}-\mathrm{XIII}{ }^{\mathrm{e}}$ siècles) "}

Eléonore Andrieu invite l'auditoire à un parcours à travers différents textes historiographiques sur l'histoire des rois francs. Dans ces textes, la présence ou l'absence d'un discours mythologique des origines détermine deux types de poétiques, liés à deux formes d'historiographie: l'historiographie chrétienne, initiée par Eusèbe de Césarée, qui gomme toute vérité politique pour élaborer une histoire du peuple 
chrétien; une historiographie héritée de l'historiographie romaine qui, à l'inverse, s'intéresse aux contingences humaines et politiques de l'histoire.

3 L'œuvre de Grégoire de Tours ( $\mathrm{vI}^{\mathrm{e}}$ siècle), premier écrivain de l'histoire des Francs, relève de la première catégorie. À l'origine de la nation franque, Grégoire évacue tout support mythique, montrant que l'incertitude est totale quant à l'identité du premier roi et à sa souche. C'est avec Clovis, premier roi chrétien, et donc seul fondateur utile de la lignée royale, que les Francs entrent dans l'histoire du christianisme. D'un point de vue stylistique, Grégoire procède par juxtaposition de cellules narratives que ne relie aucun lien causal explicite. Le lecteur doit mettre en œuvre une démarche typologique : les personnages de l'histoire ne prennent de sens que si on les rapproche de personnages bibliques. À l'appui de sa démonstration, Eléonore convoque la relation du mariage entre Clovis et Clotilde.

4 À cette poétique qui fait appel à une interprétation transcendante s'oppose la poétique de Frédégaire (VII ${ }^{\mathrm{e}}$ siècle). Celui-ci, outre qu'il intègre à son récit la légende des origines troyennes, invente un mythe qui ne sera pas repris par ses successeurs : le viol de la femme de Clodion par un monstre marin, probable père de la lignée royale. D'autre part, à l'inverse de Grégoire, Frédégaire, tissant un récit continu, se place sous l'autorité de Virgile, empruntant au poète latin le thème de l'errance, du mariage du héros avec une princesse autochtone, la fondation d'une nouvelle Troie sur la terre d'exil, etc. Ce «corsetage narratif » n'empêche pas l'amplification romanesque: du même mariage de Clovis et Clotilde dont Grégoire faisait un récit concis et exemplaire, Frédégaire fait une aventure nuptiale et politique, introduisant des personnages et des péripéties nouvelles.

5 Cette poétique, qui introduit dans les événements de l'histoire ce que Michel Zink a nommé «la bourre du quotidien», sera largement développée par la suite: la recherche de l'exhaustivité, marquée par l'invention d'anecdotes et de péripéties, l'évolution "courtoise » aussi du texte, qui se centre de plus en plus sur l'histoire d'amour de Clovis et Clotilde, sont repérables à la fois dans le Liber Historiae Francorum (VIII siècle) et dans les Grandes Chroniques de France (1274). Seul Aimoin de Fleury, à la fin $d u x^{e}$ siècle, tentera de concilier les deux poétiques de l'histoire, calquant sur un discours largement romanesque un discours chrétien de l'exemplarité.

6 À l'arrière-plan de ces deux catégories se dessinent les distinctions opérées jadis par Erich Auerbach: au discours abrupt de Grégoire, qui requiert une interprétation chrétienne ou, du moins, transcendante, s'oppose un discours intégrant la continuité temporelle et l'attention aux choses de ce monde.

7 Après cette communication, Jean-Claude Faucon revient sur la religion de Clovis, qui choisit - par conviction ou par habileté stratégique ? - le catholicisme contre l'hérésie aryenne. On interroge Eléonore Andrieu sur le lieu de fabrication des Grandes Chroniques, Saint-Denis : elle précise que Suger, conseiller du roi, inaugure une idéologie royale passablement émancipée de l'emprise du Clergé, que l'on peut rapprocher de la poétique des Grandes Chroniques. Elle est enfin amenée à préciser quelle a été la réception des textes dont elle a parlé : succès immense du Liber Historiae Francorum et des Grandes Chroniques; l'œuvre historiographique de Grégoire, considéré surtout comme un grand théologien, a en revanche été dépecée dès après sa mort. 


\section{Daniel Lacroix, « Le discours mythologique chez Saxo Grammaticus et Snorri Sturluson »}

8 Daniel Lacroix précise d'abord le contexte dans lequel s'inscrit le discours mythologique scandinave. Du fait de la tardive christianisation de l'Europe du Nord, le laps de temps est très bref entre le moment où les mythes sont liés à des croyances religieuses et celui où, repris dans un contexte chrétien, ils deviennent une matière littéraire coupée de ses origines. Pour autant, les mythes scandinaves ne passent pas abruptement d'un sens religieux païen à une élaboration littéraire : il existe déjà, avant l'évangélisation des pays scandinaves, un discours mythologique littéraire dont les clercs ont pu largement s'inspirer. Enfin, si ceux-ci s'intéressent à la mythologie, c'est avant tout pour son contenu culturel et « patrimonial ». Conscients que la Scandinavie ne peut rivaliser avec le reste de l'Europe sur le plan architectural par exemple, ils mettent en valeur une mythologie originale, en des synthèses qui rassemblent le meilleur des mythes scandinaves. Ces jalons étant posés, Daniel Lacroix examine la manière dont deux auteurs chrétiens, Saxo Grammaticus et Snorri Sturluson, intègrent ces mythes à leurs écrits.

9 Saxo a écrit autour de 1200, à la demande de l'évêque Absalon, des Gesta Danorum en seize livres dans lesquels on distingue deux parties. Les neuf premiers livres racontent l'histoire du Danemark depuis ses origines jusqu'au Ix ${ }^{e}$ siècle, moment de la conversion du pays ; les sept derniers couvrent la période qui va de l'évangélisation à l'année 1185. L'ensemble obéit à une perspective chrétienne : pour Saxo, le paganisme et ses mythes doivent être dépassés. Les modèles dont il s'inspire sont les historiographes Bède le Vénérable, Dudon de Saint Quentin, Geoffroy de Monmouth, mais aussi le poète Virgile. Aussi propose-t-il une "interpretatio romana " de la matière mythologique danoise. Les mythes, interprétés selon une perspective évhémériste, sont intégrés à une réécriture explicite de l'histoire romaine: les noms des héros sont romanisés et on exalte chez eux des vertus romaines - le courage guerrier par exemple.

Snorri, clerc islandais (1179-1241), a reçu une éducation ecclésiastique, sans être toutefois aussi marqué que Saxo par la culture latine; il n'est pas certain qu'il lisait couramment le latin, et son œuvre est entièrement rédigée en norrois. Cette culture aussi scandinave que chrétienne rejaillit sur la manière dont il réécrit les mythes scandinaves. Le Heimskringla raconte, en seize livres, l'histoire des rois de Norvège jusqu'en 1177. L'importance de la saga centrale, consacrée au roi Olaf canonisé sous le nom de saint Olaf, montre l'orientation chrétienne de l'ensemble. L'étude du premier livre se révèle particulièrement intéressante: d'un côté, Snorri développe une interprétation évhémériste de la mythologie, se livrant à une enquête étymologique pour montrer que les premiers dieux sont des hommes d'Asie jadis divinisés ; de l'autre, il exalte une forme d'héroïsme propre à la Scandinavie, insistant non pas sur les vertus guerrière des héros, mais sur leur lien à la magie ou à la poésie - leur appartenance, donc, à la première fonction indo-européenne. Ce croisement de perspectives chrétienne et scandinave distingue son œuvre de celle de Saxo.

11 Daniel Lacroix examine ensuite un autre texte de Snorri, l'Edda, rédigé une dizaine d'année avant le Heimskringla. Si le prologue, au demeurant apocryphe, reprend la thèse évhémériste, le propos du reste de l'ouvrage paraît totalement étranger à cette perspective. La première partie consiste en un long récit de l'histoire des dieux, véritable mythographie en prose, forme de la saga, insérant des poèmes en vers. 
L'auteur a probablement lu la Bible, peut-être l'Apocalypse, qui aurait pu influencer la peinture d'un "crépuscule des dieux» scandinaves. Mais il s'abstient de faire apparaître ses sources, comme pour montrer que la culture scandinave se suffit à ellemême. La suite de l'ouvrage est un traité de poétique - sans doute faut-il lire les poèmes de la première partie comme une illustration des préceptes alors développés. Daniel Lacroix conclut sur la différence de style qui distingue les deux écrivains. Au style ample et recherché de Saxo, imitant ou concurrençant la rhétorique latine, s'oppose l'économie de moyens stylistiques qui préside à la prose de Snorri, destinée à mettre en lumière l'écriture virtuose des poèmes cités. Snorri, d'autre part, fait montre d'un humour persifleur très caractéristique des sagas norroises.

\section{Valérie Fasseur, «L'énigme du Sphinx. Le début du Roman de Thèbes ou le lecteur médiéval face au signe antique »}

13 Le mythe d'œdipe, ajouté par l'auteur du Roman de Thèbes à son hypotexte (la Thébaïde de Stace), est manifestement destiné à éclairer le «sen » du roman. Le péché d'ÆEdipe serait l'événement fondateur qui expliquerait les catastrophes de la génération suivante; matrice mythologique engendrant l'écriture romanesque, il délivrerait au lecteur la conception d'un «fatum » implacable. L'épisode, qui occupe près de six cents vers, est saturé de signes, signes de paroles ou phénomènes sensibles, et invite à interroger l'attitude des différents personnages face aux signes qu'ils rencontrent.

Valérie Fasseur donne en premier lieu un exemple de signe bien compris. Analysant le dialogue au cours duquel Jocaste comprend qu'ÆEdipe a tué son mari (v. 411-422), elle montre que l'efficacité de la communication entre les deux personnages repose sur leur commune compréhension d'un double code, féodal - d'où l'échange de serments et de garanties - et privé - CEdipe, dans l'absence de haine de Jocaste, doit lire son amour.

Elle s'attache ensuite aux nombreux exemples de dysfonctionnements du signe. Laïus, au début du récit, comprend les paroles des serfs qui viennent d'abandonner ÆEdipe en fonction de ce qu'il croit savoir, sans déceler dans leurs propos un avertissement (v. 123-126). Plus tard, Edipe bafoué et traité de bâtard va consulter Apollon sur l'identité de son père (v. 171-210). Plusieurs raisons peuvent expliquer que le héros se trompe sur les paroles du dieu. D'une part Apollon, comme le précise le texte, connaît l'avenir et non le passé; il répond par conséquent à đedipe sur son futur, sans l'informer sur sa naissance. D'autre part, les paroles d'Apollon opèrent une disjonction entre l'homme que tuera CEdipe et son père (v. 204-206). Cette disjonction correspond au regard qu'ÆEdipe portera sur les faits, la révélation de l'identité du mort se plaçant après le meurtre. L'erreur d'€Edipe consiste donc à ne pas voir dans l'oracle un reflet de sa subjectivité. Cette méprise l'engage à négliger les paroles d'Apollon (il " n'ot cure ", v. 210), puis à les oublier, la quête du père et le souvenir de l'oracle disparaissant par la suite du récit.

16 À cette scène, Valérie Fasseur oppose celle de l'énigme de Pyn (v. 267-374), qu'đedipe résout précisément en se reconnaissant dans les paroles du monstre : dans un cas il se sauve en se reconnaissant (v. 331-358), dans l'autre il se perd en s'ignorant lui-même. Toutefois, même dans l'épisode de l'énigme, son attitude ne lui permet d'accéder qu'à un salut partiel et provisoire. La dimension cyclique de la vie, que traduit le nombre de 
« piez» utilisés par l'homme - quatre, puis trois, puis deux, puis trois, puis quatre évoque l'image de la roue de Fortune, mentionnée plus haut dans le texte (v. 130). Cette image est aussi celle du destin d'Ædipe, appelé à tomber au faîte de sa gloire ; cela, Ædipe se montre incapable de le comprendre. L'explication de ses méprises réside sans doute dans le fait que ne connaissant pas sa véritable identité, il est, dans deux cas, inapte à se reconnaître dans les signes qui jalonnent sa route.

Valérie Fasseur revient enfin sur la relation qui unit CEdipe et Jocaste, remarquant que celle-ci, dès sa rencontre avec đEdipe, a tous les éléments en main pour reconnaître dans l'étranger son fils : connaissant le premier oracle d'Apollon, elle sait que son fils, meurtrier de Laïus, deviendra son époux. Si elle ne prévoit pas, au moment de son dialogue avec EEdipe (v. 411-422), leur inceste futur, si elle ne « voit » les pieds meurtris d'ÆEdipe qu'au bout de vingt ans de vie commune, c'est qu'elle ne lit dans chaque signe que ce qui lui permet d'assouvir son désir. Or le récit tout entier insiste sur le danger des affects, qui brouillent les facultés d'analyse des hommes. L'auteur, à la conception antique du "fatum», substitue l'idée que les personnages se perdent eux-mêmes à cause de leurs passions.

18 Ainsi, pour lire adéquatement un signe, il faut d'abord l'identifier comme tel, puis l'interpréter, le signe délivrant toujours un message brouillé; pour l'interpréter correctement, il faut se libérer de ses affects. Ce dernier point pose le problème de la liberté du personnage face à l'oracle: celui-ci est-il une fatalité implacable ou une puissance douée de prescience, mais qui ne pèse pas sur le destin des hommes? Laïus, en considérant le tout premier oracle comme une fatalité, engendre une cascade de crimes: sa conception du signe est manifestement erronée. Les oracles mettent les hommes face aux signes sans pour autant leur ôter leur liberté. L'auteur laisse transparaître une conception chrétienne de la divinité : Dieu sait tout, mais l'homme garde son libre-arbitre. Pour finir, Valérie Fasseur ébauche un parallèle entre la situation des personnages face aux signes et celle du lecteur face au texte, réseau de signes proposés à l'interprétation.

19 Au cours de la discussion, deux aspects sont abordés. Eléonore Andrieu revient sur la question du "fatum", qui revêt dans l'historiographie ecclésiastique un caractère inéluctable, véritable fatalité présidant à la trajectoire des rois et des héros. Si cette impression était vérifiée, elle permettrait de dessiner une ligne de partage entre l'historiographie médiévale et le roman antique. Puis Jean-Claude Faucon insiste sur le fait qu'đEdipe, lorsqu'il rencontre Jocaste, manifeste un aveuglement similaire à celui de sa mère ; à côté de la condamnation du désir de Jocaste, il y a une condamnation de la vaine gloire que recherche ÆEdipe, prêt à se voiler les yeux sur l'identité de sa mère pour accéder au trône. Pour Valérie Fasseur, cette remarque est liée à la question des affects: Jocaste est une incarnation de la concupiscible tandis qu'Édipe, qui agit plusieurs fois sous l'emprise de la colère, représente l'irascible, les deux personnages étant ainsi gouvernés par des élans qui échappent à toute rationalité.

\section{Cristina Noacco, « Lire Ovide au XII siècle : Arnoul d'Orléans commentateur des Métamorphoses »}

Dans le dernier quart du XII ${ }^{e}$ siècle s'affrontent, à Orléans, deux approches divergentes d'Ovide: celle de Matthieu de Vendôme, qui cherche à transposer en "roman" la culture latine; la glose interprétative d'Arnoul d'Orléans, qui vise à soulever 
l'«involucrum» des textes antiques. Cristina Noacco se propose d'analyser l'interprétation des Métamorphoses par Arnoul d'Orléans et l'évolution des lectures interprétatives du mythe de Narcisse.

Elle commente d'abord l'accessus d'Arnoul d'Orléans aux Métamorphoses d'Ovide. À partir des gloses de Guillaume de Conches au Timée de Platon et de la Consolation de Philosophie de Boèce, l'auteur infléchit le sens du texte ovidien dans une direction nettement morale et spirituelle. L'accessus donne ainsi un contenu chrétien à la métamorphose. Aussi Arnoul, dans la suite de l'ouvrage, développera-t-il après chaque métamorphose des interprétations allégoriques, morales ou historiques.

Le mythe de Narcisse, utilisé chez Arnoul pour fustiger l'orgueil et pour montrer combien les choses de la terre, ici représentées par la fleur, sont vaines et inconstantes, fera l'objet, entre le $\mathrm{XII}^{\mathrm{e}}$ et le $\mathrm{xIV}^{\mathrm{e}}$ siècle, de diverses interprétations. Après avoir brièvement analysé le Lai de Narcisse (fin $\mathrm{du}_{\mathrm{XII}}{ }^{\mathrm{e}}$ siècle), dont la morale rappelle les gloses des grammairiens sans que l'on puisse toutefois prouver sa filiation avec les textes didactiques, Cristina Noacco s'attache à deux lectures plus nettement tributaires de l'ouvrage d'Arnoul : les Integumenta Ovidii de Jean de Garlande (xiII ${ }^{\mathrm{e}}$ siècle), où la fleur symbolise la caducité des choses terrestres, et l'Ovide moralisé (début du XIV ${ }^{e}$ siècle), qui condamne l'orgueil et la vanité de Narcisse et où la fleur, encore, dit la fragilité des choses de ce monde.

\section{Anne-Marie Colin, « Piramus et Tisbé : la réécriture d'un mythe païen »}

Anne-Marie Colin se propose d'étudier la manière dont l'auteur de Piramus et Tisbé, pièce de 922 vers rédigée entre 1155 et 1160 , réécrit le récit mythologique de Pyrame et Thisbé présent au Livre IV des Métamorphoses d'Ovide (v. 55-166). La communication porte d'abord sur le devenir de la composante mythique du récit, à savoir la métamorphose, puis sur la « religion » de chacun des deux textes.

Anne-Marie Colin commence par préciser le sens à donner au terme "mythe». Sur le plan du contenu, le mythe, selon Mircea Eliade, raconte une histoire sacrée ayant eu lieu dans un temps primordial ; il raconte comment la réalité est advenue, réalité totale du cosmos ou réalité fragmentaire. L'aspect mythique est ainsi défini par la notion d'étiologie. Dans le récit d'Ovide, la portion de réalité dont rend compte le mythe est la couleur noire des mûres. A ce contenu correspond un critère formel, l'emploi de temps spécifiques à la narration du mythe : récit au passé, conclusion au présent de vérité générale. Ce critère s'accompagne de deux autres types de signaux, métalinguistiques Ovide, au Livre I (v. 1-4), annonce que son objet sera de conter les métamorphoses opérées par les dieux - et situationnels - une mise en scène du récit, ici raconté à un auditoire par la fille de Minyas.

Après avoir ainsi défini les contours du mythe et démontré le caractère mythique du récit ovidien, Anne-Marie Colin montre que l'objet étiologique du récit s'efface dans la réécriture médiévale. L'auteur, à la différence d'Ovide, omet de préciser la couleur d'origine des mûres : les « fruits de neige » d'Ovide laissent place à un «morier » dont on ne mentionne pas les fruits. Plus loin, la description du moment où les mûres noircissent fait l'objet de deux investissements très différents. Ovide, avec une précision toute réaliste, explique comment le sang de Pyrame abreuve les racines de 
l'arbre et décrit le jaillissement de son sang éclaboussant les fruits; la comparaison avec un tuyau percé assimile ce jaillissement à un mécanisme physique. À cette description toute concrète, l'auteur médiéval substitue une métamorphose d'origine miraculeuse, réponse des dieux à la prière de Piramus (v. 771-792). Quelques vers plus loin, le vœu de Thisbé, dans le texte ovidien, porte explicitement sur la couleur des mûres; au contraire le mûrier, dans Piramus, est pris dans une énumération qui minimise son importance («Lune, fontaine, prez, moriers, / Nuit pale... », v. 854-859). La conclusion étiologique d'Ovide enfin subit une radicale transformation, la mention du mûrier disparaissant complètement du récit médiéval (v. 920-921). La vocation étiologique du récit est ainsi remplacée par la démonstration des liens qui unissent l'amour et la mort.

Anne-Marie Colin examine ensuite la "religion » des deux récits, antique et médiéval. Dans le texte ovidien, la présence des dieux est pour le moins ténue : la prière de Thisbé s'adresse uniquement à l'arbre, la métamorphose a des causes naturelles; les dieux, certes, apparaissent dans la conclusion, mais ils sont mis sur le même plan que les pères des deux héros. Les dieux, si du moins ce sont eux qui font la métamorphose, sont assimilés à une force de la nature, ce qui correspond à l'orientation naturaliste d'Ovide. À cette quasi-absence de la divinité s'oppose son omniprésence dans le récit médiéval. Même en laissant de côté les manières de parler proprement médiévales (exclamations ou invocations formulaires à Dieu ou aux Dieux), on relève de nombreux vers faisant intervenir une instance transcendante, sous deux formes différentes. D'une part, l'auteur médiéval ajoute à l'hypotexte deux divinités païennes, Vénus et le «Diex d'amour ", se livrant à une manifeste paganisation du récit ovidien. En même temps, ces prières aux dieux constituent des réflexes chrétiens, comme, au demeurant, le fait que l'auteur ne puisse penser la métamorphose que sur le mode du miracle. Ces indices d'une conscience chrétienne sont confirmés par deux passages : la prière par laquelle Piramus demande au "peres qui mains en haut» d'éteindre en lui les flammes de l'amour (v. 187-188) ; la prière de Tisbé à un " Diex grans ", plus loin invoqué comme un «Diex pere », dans lequel on reconnaît sans peine le dieu chrétien (v. 521-529). La paganisation que l'auteur imprime au récit ovidien est par conséquent une paganisation «de surface ", et dissimule mal la conscience chrétienne qui préside à la réécriture du mythe.

Anne-Marie Colin conclut sur l'idée que l'auteur médiéval substitue, au mythe d'origine de la mûre, le mythe, cette fois pris au sens de récit exemplaire, d'un amour passionné conduisant à la mort.

$\mathrm{Au}$ cours de la discussion, Cristina Noacco revient sur la définition du mythe, que l'on peut aussi définir par la présence de mythèmes, puis sur l'image de l'amour dans Piramus et Tisbé, plutôt amour innocent que passion. Anne-Marie Colin s'accorde avec cette remarque et propose, pour cerner le "genre» de Piramus et Tisbé, la notion d'idylle tragique. Une autre assistante fait observer que le mur, paradoxalement, est autant un moyen de communication qu'un obstacle - le moment où les deux amis se parlent à travers la muraille est même celui où ils communiquent le mieux. Cristina Noacco revient encore sur la définition de l'amour dans le récit médiéval, engageant une discussion qui se prolongera au moment de la pause. 


\section{Danielle Quéruel, « Jason et Médée dans les textes médiévaux ( $\mathrm{XII}^{\mathrm{e}}-\mathrm{XV}^{\mathrm{e}}$ siècles)"}

Jason et Médée forment un couple exemplaire d'une histoire passionnelle complexe et torturée. Leur histoire connaîtra, au cours des siècles, des fortunes diverses, Jason oscillant entre l'image magnifiée du conquérant et celle de l'infidèle, Médée entre celle de la sorcière et celle de la victime - transmise notamment par les Héroïdes d'Ovide. Quant à Jason, sa réputation se trouve ternie dès les Argonautiques d'Apollonios de Rhodes (III siècle av.J.-C.). Danielle Quéruel propose de parcourir différents textes médiévaux afin d'examiner les fortunes, bonnes ou mauvaises, de Jason et Médée.

Le premier texte en langue vernaculaire qui relate leur histoire, fondateur à bien des égards, est le Roman de Troie, rédigé vers 1160 par Benoît de Sainte-Maure. Au seuil du roman, l'auteur s'arrête sur l'histoire du couple. Son récit met en cause l'ingratitude de Jason vis-à-vis de Médée, préfigurant la perfidie des Grecs qui, plus tard, auront recours à la ruse pour vaincre les Troyens. Cette faute première revêt ainsi une valeur explicative. D'autre part, le récit omet à la fois Hypsipyle, la première amante de Jason, et Creüse, sa seconde femme, se resserrant sur la seule Médée.

31 Ce texte, dans lequel Jason reçoit une image fortement négative, connaîtra une grande postérité. Dans le Roman de la Rose, Jean de Meun énumère les bienfaits de Médée et critique sévèrement Jason, un «faus » qui «sa foi li manti» (v. 13201). À sa suite, Eustache Deschamps, puis Christine de Pisan dans la Mutacion de Fortune (v. 14765-14786), dressent un véritable réquisitoire contre le déloyal Jason.

32 À l'opposé de cette tradition, plusieurs textes s'attachent, de différentes manières, à réhabiliter la figure de Jason. Evrart de Conty (ca.1330-1405), dans le Livre des Eschez amoureux moralisés, compare point par point le parcours de l'amant dans le jardin d'amour avec l'expédition de Jason en Colchos: Jason, débarrassé par la fiction allégorique de sa perfidie, recouvre une stature de conquérant. En un sens non plus courtois, mais chrétien, l'Ovide moralisé fait de Médée l'image de la tentation et des aventures de Jason la métaphore d'une conquête du Bien. Enfin, à la suite de la création, en 1430, de l'Ordre de la Toison d'or, on assiste à des réinvestissements divers de l'histoire de Jason. Représentation théâtrale de son histoire lors du Banquet du Faisan, où des acteurs miment ses aventures dans une salle tapissée de scènes de la vie d'Hercule; Médée disparaît complètement de l'intrigue. Réhabilitation romanesque, surtout, dans l'Histoire de Jason de Raoul Lefèvre (ca. 1460). Contrairement à Benoît de Sainte-Maure, dont le récit évinçait toute autre femme que Médée, Raoul multiplie les figures féminines. Il invente à Jason un premier amour, Mirro, reine d'olyferne, n'oublie par le séjour à Lemnos où Jason engrosse Hypsipyle, puis fait de la passion entre Jason et Médée le fruit d'enchantements magiques qui effacent chez Jason le souvenir de Mirro. Jason est blanchi de toute culpabilité : fidèle toujours à Mirro, poussé contre son gré à s'unir à la reine de Lemnos, pris, malgré lui toujours, dans les lacs de Médée, marié enfin à Mirro après la mort de Creüse, il revêt à nouveau une image entièrement positive. Parallèlement, Médée apparaît en sorcière inquiétante, coupable de meurtres sanglants, sauvée in extremis à la fin du roman quand Jason, ayant perdu successivement toutes ses femmes, la retrouve dans une forêt, errante et repentie, et l'épouse une seconde fois. 


\section{Florence Bouchet, « Le clerc, le poète et le fou : de quelques discours possibles sur Narcisse à la fin du Moyen Âge »}

33 La fin du Moyen Âge est marquée par une attitude très réflexive à l'égard de la mythologie. De vifs débats opposent les clercs, par exemple, quant à la culpabilité de Narcisse, dont l'histoire reçoit plusieurs interprétations, courtoise - Narcisse a cédé à l'amour - néo-platonicienne, sous l'influence de Plotin - Narcisse se laisse prendre par une vaine image - ou morale - Narcisse est l'image de l'orgueilleux. L'objet de Florence Bouchet est de présenter quelques discours sur Narcisse aux derniers siècles du Moyen Âge.

Evrart de Conty, dans le Livre des Eschez amoureux moralisés, développe une réflexion sur l'amour à partir notamment de figures mythologiques; la fontaine de Narcisse fait partie des dangers du jardin d'amour. Evrart en donne deux interprétations. Une interprétation mondaine: Narcisse représente les jeunes gens du monde, Echo les femmes séductrices; une interprétation évhémériste: Echo, jadis, aima Narcisse; Narcisse, de son côté, fut amoureux d'une cruelle qui le fit mourir d'amour. À la suite de ces deux interprétations, Evrart livre au lecteur quelques leçons complémentaires : la gloire mondaine est vanité, l'amour loyal dure jusqu'à la mort, il faut éviter les amours impossibles, la vue est pour déclencher la passion plus puissante que l'ouïe... L'auteur décompose le mythe en plusieurs éléments assimilables à des expériences ordinaires, ce que montre l'usage du comparatif "comme». Il s'en tient à une explication naturaliste et rationaliste de l'amour (l'adverbe "raisonnablement » en témoigne), laissant de côté toute leçon spirituelle et chrétienne. Le mythe de Narcisse lui permet avant tout de définir les conditions de viabilité de l'amour.

Jean Froissart (ca.1337-1404), dans le Joli Buisson de Jonece, propose une nouvelle réécriture du mythe. Narcisse ne s'est pas remis de la mort d'Echo, sa bien-aimée ; il voit dans l'eau le visage de la morte. L'illusion visuelle est renforcée par le phénomène acoustique de l'écho, qui « répond » aux plaintes de Narcisse. Le héros enfin dépérit et trépasse. La version donnée par Froissart constitue une invention consciente : Froissart connaît parfaitement la version ovidienne, qu'il cite ailleurs « correctement ». L'auteur semble avoir à l'esprit l'histoire d'Actéon, Narcisse poursuivant un cerf avant de parvenir à la fontaine. La réécriture permet de présenter Narcisse comme un double malheureux du narrateur.

Enfin l'Istoire de Narcissus et de Echo, pièce de théâtre anonyme, respecte globalement la trame du mythe, mais introduit la figure moralisatrice et railleuse $d u$ fou, parole de vérité dans le théâtre médiéval. Sa présence offre d'abord un contre-point comique, voire farcesque, au sérieux convenu du mythe. Dans un premier passage (v. 203-218), le fou, commentant les plaintes d'Echo, les présente comme une parodie de dispute scolastique (Echo argue "pro et contra", v. 216); mimant le triangle amoureux du fabliau, il fait comme si l'indécision d'Echo portait sur le choix d'un amant, Narcisse ou lui-même ; il feint de voir en Echo une femme échauffée par la luxure. Il traite enfin Narcisse de noms peu glorieux (v. 937-942, « maistre penart ", « cornart ») et l'englobe dans la catégorie des «folz» (v. 1103), renversant les rôles du drame. Ses interventions opèrent ainsi un détournement parodique du mythe et manifestent une étonnante - et réjouissante ! - aptitude à la distanciation. 

diffuse du poème d'Alain Chartier, la Belle Dame sans Merci (1424). Echo supplie Narcisse d'avoir d'elle «mercy » (v. 547), le fou reproche à Narcisse la " grant durté » dont il fait preuve (v. 854). La référence, dans les propos du fou, est encore plus explicite : le fou propose de nommer Narcisse « sans mercy » comme la dame du même nom (v. 571-580) puis, à la fin de la pièce, enjoint au public de se garder de suivre l'exemple du héros ou de la "Dame sans mercy» (v. 1264-1269). Narcisse, qualifié par le biais de ce nouveau mythe qu'est la Belle Dame sans Merci, incarne une forme de revanche masculine sur la dame d'Alain Chartier. L'ensemble traduit une conception désabusée de l'amour courtois, miroir d'un monde où l'amour est désenchanté et les amants, faux et cruels.

Le mythe, fait de bricolages - au sens que Levi-Strauss donne à ce terme -, convenait bien aux pratiques d'écriture médiévales. Parce que les $\mathrm{XIV}^{\mathrm{e}}$ et $\mathrm{XV}^{\mathrm{e}}$ siècles se sont beaucoup interrogés sur l'ambiguïté des signes, le mythe de Narcisse, avec la double présence de reflets visuels et sonores, offrait aux auteurs une matière privilégiée.

De l'écriture du mythe à sa réécriture, de sa réécriture à sa glose, des glissements s'opèrent, dont rend compte cette journée d'étude sur le discours mythologique. Comme l'ont montré les participants, le mythe revêt au Moyen Âge plusieurs fonctions possibles. Sur un plan narratologique, il peut être, au seuil de l'œuvre, une matrice engendrant le récit ; E. Andrieu l'a montré pour l'écriture de l'histoire, V. Fasseur et D. Quéruel ont évoqué ce rôle pour deux romans antiques, le Roman de Thèbes et le Roman de Troie. Les réécritures dont il fait l'objet manifestent la manière dont les divers courants de la pensée médiévale ont pu récupérer et adapter ses figures. Mythes romanisés, imprégnés de culture latine, chez Saxo Grammaticus par exemple, dont les héros sont dotés de noms latins et de vertus romaines (D. Lacroix), ou chez Frédégaire, qui réinvestit le modèle virgilien (E. Andrieu); mythes christianisés, relus à la lumière de la pratique exégétique, donnant lieu à des interprétations évhéméristes, morales ou allégoriques, dans de nombreuses œuvres (D. Lacroix, C. Noacco, F. Bouchet). Quand l'allégorie se fait courtoise et non plus religieuse, c'est un nouveau sens qui paraît, mondain plutôt que «célestiel », et parfois un nouveau mythe, proprement médiéval celui-là, qui émerge : mythe de l'amour conduisant à la mort, mythe de la Belle Dame sans Merci - à condition de prendre le mot «mythe " dans l'acception de "récit exemplaire" (A.-M. Colin, F. Bouchet). Au milieu de tant de textes où le mythe se trouve chargé d'une signification étrangère à ses origines, la démarche de Snorri Sturluson, qui recueille, sans glose ni jugement, les mythes scandinaves, n'en apparaitt que plus originale - folkloriste ou, du moins, mythographe avant l'heure ? Le discours mythologique oscille ainsi entre la fidélité et l'écart, gommant ou affirmant l'exotisme du mythe, le ramenant au Même ou le rejetant dans une altérité de rites et de croyances, en des remodelages dont le sens, au fil des auteurs et des siècles, est sans cesse renouvelé. 
INDEX

Keywords : speech, mythology, literature

Mots-clés : discours, mythologie, littérature 